\title{
Pregabalin: Dose-Response Relationship in Generalized Anxiety
}

Disorder

Mark J. Boschen

Senior Lecturer in Clinical Psychology, School of Psychology and Griffith Health Institute, Griffith University, Parklands Drive, Southport Qld 4215, Australia. Ph: +61 7 55528283. Fax +61 7 55528291. email: m.boschen@ griffith.edu.au

Running Title: Dose-Response in Pregabalin for GAD 


\begin{abstract}
Introduction: Pregabalin is a structural analogue of the inhibitory neurotransmitter GABA. Although pregabalin has demonstrated efficacy in the treatment of GAD in a number of randomized controlled trials, there is little published data on the optimal dose for treating this condition. The objective of the current study was to examine the doseresponse relationship for treatment of generalized anxiety disorder with pregabalin.
\end{abstract}

Method: The current study pools results from previous fixed-dose treatment trials of GAD with pregabalin and uses curve-fitting statistical procedures to generate curvilinear regression lines as a synthesis of previous dose-response information.

Results: Peak reduction in anxiety symptoms was observed to occur at a dose of approximately $450 \mathrm{mg} /$ day, with a small reduction in treatment effect beyond this dose. Treatment effect on psychic anxiety symptoms reached a peak at approximately 400mg, with a reduction in efficacy beyond this dose. Somatic anxiety symptoms showed continued increase in therapeutic effect up to the maximum dose of $600 \mathrm{mg} / \mathrm{day}$.

Discussion: Implications for psychiatric practice, limitations of the methodology and avenues for future research are discussed.

Key Words: Pregabalin, Generalized Anxiety Disorder, Anxiety Disorder, Anxiety, Dose-Response Curve 


\section{Introduction}

Generalized anxiety disorder (GAD) is a chronic mental disorder in which the individual experiences significant difficulty controlling excessive worry and anxiety. Other symptoms such as restlessness, being easily fatigued, problems with concentration, excessive muscle tension, irritability, and insomnia may also be present. To meet DSM-IV criteria for GAD, an individual must experience these symptoms over at least six months, and they must lead to clinically significant functional impairment or distress. $^{[1]}$ Lifetime prevalence of GAD is estimated at $6.2 \% .^{[2]}$ GAD impacts significantly on quality of life, life satisfaction, and general well-being, leading to significant functional impairment and increased utilization of health services. ${ }^{[3-6]}$ Research into GAD has increased over the last 25 years $^{[7]}$, but despite the existence of effective treatments, there remains a need for further research into treatment for the condition to reduce problems such as relapse. ${ }^{[8,9]}$

Pregabalin is a structural analogue of the inhibitory neurotransmitter $\gamma$ aminobutyric acid (GABA). ${ }^{[10,11]}$ In contrast to earlier anxiolytic medications such as the benzodiazepines, pregabalin does not bind with the post-synaptic GABA receptor complex, instead exerting its effect through reducing calcium ion flow through presynaptic calcium ion channels by binding with the $\alpha_{2} \delta$ subunit of pre-synaptic voltage-dependent calcium channels. ${ }^{[12-14]}$

Pregabalin has been demonstrated to be efficacious for the treatment of GAD over a series of eight published trials. ${ }^{[15-22]}$ and one meta-analysis. ${ }^{[23]}$ Initial evidence also suggests that pregabalin is effective in treating GAD in older adults, ${ }^{[20]}$ and reducing the potential for relapse. ${ }^{[22]}$

Although recommended dose ranges for pregabalin in the treatment of GAD range from $150 \mathrm{mg}$ to $600 \mathrm{mg}$ per day, in two or three divided doses, the literature on the 
specific dose-response relationship between pregabalin dose and reduction in GAD symptoms has not been fully elucidated. Such information can aid prescribers in considering whether the potential for increased side effects often seen with higher doses is justifiable when contrasted with the probability of increased therapeutic effect.

Two previous studies have examined the dose-response relationship of pregabalin in the treatment of GAD. In the first of these ${ }^{[24]}$, data was pooled from four previous randomized controlled trials ${ }^{[15-18]}$ to examine the optimal dose of pregabalin for treatment of anxiety symptoms, as well as GAD specific anxiety symptoms. In this study the authors used effect size statistics as their measure of treatment effect. The results of this analysis led the authors to suggest that doses up to $150 \mathrm{mg}$ were clearly insufficient for the treatment of GAD, but that a plateau in response is observed above this dose, meaning that doses between 200 and $600 \mathrm{mg} /$ day show similar responses.

A more recent analysis ${ }^{[25]}$ examined the effect of pregabalin on anxiety symptoms across five published ${ }^{[15-19]}$ and one unpublished trial. Although these authors did not apply a curve fitting procedure to their data, they reported that for overall anxiety symptoms, a dose of $300 \mathrm{mg} /$ day of pregabalin was associated with optimal response. When the authors examined the psychic anxiety and somatic anxiety subscales of the Hamilton Anxiety Rating Scale (HAM-A) separately, they found that low doses demonstrated a therapeutic effect on the HAM-A psychic anxiety factor (anxious mood, tension, fears, insomnia, cognitive symptoms, depressed mood, behaviour at interview), but not on the somatic anxiety measure (somatic muscular, somatic sensory, cardiovascular, respiratory, gastrointestinal, genitourinary, and autonomic symptoms). These results reinforce the need to examine not only overall anxiety symptoms, but also subgroups of these symptoms, to examine the effect of pregabalin on different symptoms at different doses. 
The current study extends on previous research examining the dose-response relationship of pregabalin on GAD symptoms in three ways. Firstly, it is based on a recent search of the available published literature, and considers a number of studies that were not available to earlier authors. Secondly, it used curve-fitting statistical techniques to provide a mathematical model of the dose-response relationship, and assessed the goodness-of-fit of these regression curves. Thirdly, it examines separately the dose-response curve for somatic and psychic anxiety symptoms, in addition to overall anxiety symptoms.

\section{Methods}

In June 2011, a literature search was conducted using the PubMed database of medical/health literature to identify double-blind, randomized controlled trials for inclusion in the analyses. The search term used was "PREGABALIN AND (GAD OR ANXIETY)", ensuring that any articles relating to GAD or other anxiety disorders were likely to be selected in the first round. The list of 186 returned articles was edited for relevance, and then the reference sections of each of the articles retrieved were examined for additional research papers that may be relevant to the study.

To be included in the analysis, a study was required to be a published report, using original data, on the efficacy of pregabalin in treating individuals with a primary diagnosis of GAD. Included studies were required to use the HAM-A as the primary outcome measure, and involve a study duration of four to eight weeks. Assignment of patients in each included study was required to be random. Doses of pregabalin were required to be fixed over the course of the trial, and outcome measures were required to be presented for all participants. 
A total of eight randomized controlled trials were considered for analysis ${ }^{[15-22]}$. Two were excluded for using variable dosages of pregabalin during the treatment phase. ${ }^{[20,21]}$ One was excluded as it presented change scores on the HAM-A only for treatment responders. ${ }^{[22]}$ Table 1 presents the excluded studies, along with the reason for their exclusion. Table 2 presents the studies included in the analyses.

Using the five studies which met inclusion criteria, the administered fixed dose of pregabalin was plotted against the mean reduction in symptoms as measured by the HAM-A total score, as well as the psychic anxiety and somatic anxiety subscales. Placebo response rates were plotted as a pregabalin dose of 0mg. Curve fitting procedures were conducted using SPSS version $17^{[26]}$ to give both a graphic representation of the line of best fit through the datapoints, as well as an analysis of the statistical significance of the derived regression line.

\section{Results}

The plot of pregabalin dose against reduction in total HAM-A scores for the five included studies is shown in Figure 1. The quadratic line of best fit through the datapoints from the different studies was significant, with pregabalin dose accounting for $55.4 \%$ of the observed variance in symptom reduction (see Table 3). Symptom reduction increased gradually from a placebo dose up to approximately 450mg/day, with reduced effect at the maximum recommended dose of $600 \mathrm{mg} / \mathrm{day}$.

A quadratic dose-response curve was also the line of best fit through the data for the four studies which provided data on the mean reduction in HAM-A psychic anxiety subscale. In this dose-response curve, pregabalin dose accounted for $42.7 \%$ of treatment response. This line showed a similar pattern of a rise in treatment response 
from placebo, followed by a peak (this time at approximately $400 \mathrm{mg} / \mathrm{day}$ ), and then a gradual reduction in efficacy as doses approached $600 \mathrm{mg} /$ day. The statistical parameters of the regression line are presented in Table 3, and the dose-response curve plot is shown in Figure 2.

For the HAM-A somatic anxiety subscale, the dose-response curve findings were more complex. A quadratic function was attempted, and although this accounted for $40.8 \%$ of variance in symptom reduction variance, it failed to reach statistical significance $(p=0.056)$. Better fit to the data was sought by examining a range of other potential functions. The best fit was found with an exponential function, which accounted for $28.7 \%$ of treatment response, and was statistically significant (see Table 3). The quadratic function again showed a peak in treatment response at around $400 \mathrm{mg} /$ day, while the exponential function suggested that treatment response on somatic symptoms may continue to increase up to the maximum dose of $600 \mathrm{mg} / \mathrm{day}$.

\section{Discussion}

The dose-response curve presented here suggests that while low doses of pregabalin are associated with a significant decline in clinician-rated anxiety symptoms, there is additional benefit gained by increasing the dose up to 450mg per day. Beyond 450mg, however, the reduction in anxiety symptoms does not continue to improve, and may even begin to reduce slightly as the dose approaches $600 \mathrm{mg} / \mathrm{day}$. A similar doseresponse curve shape was found for psychic anxiety symptoms, but with an earlier peak response at around 400mg. In contrast, psychic anxiety symptoms may show continued improvement beyond these mid-range dosages, up to the maximum dose of $600 \mathrm{mg} / \mathrm{day}$. 
The reason for the different dose-response curves seen in the psychic anxiety and somatic anxiety subscales is unclear, but may have important clinical implications. The psychic anxiety symptoms of the HAM-A are anxious mood, tension, fears, insomnia, depressed mood, and behaviour at interview. The somatic anxiety subscale is comprised of somatic (muscular), somatic (sensory), cardiovascular, respiratory, gastrointestinal, genitourinary, and autonomic symptoms. It may be the case that the action of pregabalin to reduce psychic and somatic anxiety symptoms occurs in different neural pathways/circuits, decoupling their response to pregabalin. Circuits which underlie the somatic symptoms may experience a maximum response to pregabalin at a moderate dose, while those underlying somatic anxiety symptoms may benefit from maximal doses. This hypothesis, however, would require considerable further investigation.

There may be significant clinical implications of these different dose-response curves for somatic and psychic anxiety symptoms. Dosages of the drug may be tailored to the specific symptoms of the patient, with higher doses being used in patients with predominantly somatic symptoms. In contrast, patients with GAD where psychic symptoms are most prominent may not experience additional benefit from higher doses.

The current results accord with those found in an earlier dose response study which used a smaller number of studies in its analysis. ${ }^{24}$ Bech reported that the doseresponse curve for pregabalin on HAM-A scores in GAD reached a peak somewhere between $200 \mathrm{mg}$ and $450 \mathrm{mg} /$ day, with no additional effectiveness at $600 \mathrm{mg} / \mathrm{day}$.

While these results present some suggestion that moderate doses of pregabalin may be associated with greatest reduction in anxiety symptoms, high doses (i.e., those above $450 \mathrm{mg} /$ day) may not provide additional benefits, but still expose the patient to higher risk of adverse events. In previous research of pregabalin in the treatment of 
GAD, adverse events have been higher on a dose of $600 \mathrm{mg} /$ day compared with lower doses. ${ }^{27}$ Adverse events such as dizziness and somnolence may also make independent contributions to functional impairment if they are severe and/or prolonged.

Furthermore, the adverse events experienced by those taking pregabalin significantly contribute to dropout. ${ }^{28}$ Individuals on higher doses may therefore have increased risk of impairment and dropout from side-effects, without additional therapeutic benefit.

Several limitations should be acknowledged in the current analysis. Firstly, the dose-response curves presented herein are based on a comparatively small number of five studies that met the inclusion criteria, while the results for the somatic and psychic anxiety subscales are based on only four studies which presented subscale data. This small number of studies provides a small dataset on which to base the dose-response curves. As further studies of the efficacy of pregabalin are published, the results of these should be used to re-examine the dose-response curve presented here.

The datapoints used to generate the dose-response curve here were the group means for different cohorts treated with pregabalin or a pill placebo. An analysis similar to the one presented here could make use of individual doses and response rates to examine whether dose-response curves were similar to those in Figures 1 to 3.

A further limitation is that our data sources only examine the acute effects of pregabalin administration over periods of between four and eight weeks. It is possible that higher doses may be useful in treating refractory cases of GAD, and that this would not be apparent using our analysis methods. On a related note, our study pooled analysis that used different treatment durations, ranging from four to six weeks. While these would both be considered to represent acute treatment of GAD with pregabalin, we are unable to rule out whether these different time periods may have introduced a complicating factor into our analysis. 
The current study examined the dose-response curves for total and subscale total scores on the HAM-A. Although these scales have been factor analytically derived and psychometrically validated, they may mask differences in changes in severity of individual GAD symptoms. Future research may benefit from examination of the effect of different doses of pregabalin on individual GAD symptoms or HAM-A items.

The dose-response curve presented here is presented as an additional contribution to the information regarding the therapeutic effects of pregabalin in GAD. It is hoped that future research can examine individual dose-responses, and make use of larger samples of studies. More investigations of pregabalin over the longer term, may also allow analyses to incorporate duration of treatment into the understanding of optimal use of the drug.

\section{Conclusion}

Generalized anxiety disorder is a chronic, impairing anxiety disorder. Pregabalin is a newer anxiolytic which has demonstrated efficacy across eight published trials. When the relationship between pregabalin dose and treatment effect on anxiety symptoms is examined, the dose-response curve shows a peak response at a dose of approximately $450 \mathrm{mg}$ of pregabalin. Greatest reduction in psychic anxiety symptoms is seen at approximately $400 \mathrm{mg}$, while there is some evidence that response of somatic anxiety symptoms may continue to increase up to the maximum dose of $600 \mathrm{mg}$. The increased risk of side effects and dropout from higher doses, as well as the results presented here, should be considered before prescribing doses above this optimal range. 


\section{Reference}

${ }^{1}$ American Psychiatric Association. Diagnostic and Statistical Manual of Mental Disorders $4^{\text {th }}$ ed. text rev. Washington, DC: American Psychiatric Press, 2000

${ }^{2}$ Somers JM, Goldner EM, Waraich P, Hsu L. Prevalence and incidence studies of anxiety disorders: A systematic review of the literature. Can J Psychiatry 2006; $51: 100-113$

${ }^{3}$ Barrera TL, Norton PJ. Quality of life impairment in generalized anxiety disorder, social phobia, and panic disorder. J Anxiety Disord 2009; 23:1086-1090

${ }^{4}$ Stein MB, Heimberg RG. Well-being and life satisfaction in generalized anxiety disorder: Comparison to major depressive disorder in a community sample. $J$ Affect Disord 2004; 79:161-166

${ }^{5}$ Kroenke K, Spitzer RL, Williams JBW, Monohan PO, Löwe B. Anxiety disorders in primary care: Prevalence, impairment, comorbidity, and detection. Ann Intern Med 2007; 146:317-325

${ }^{6}$ Jones GN, Ames SC, Jeffries SK, Scarinci IC, Brantley PJ. Utilization of medical services and quality of life among low-income patients with generalized anxiety disorder attending primary care clinics. Int J Psychiatry Med 2001; 21:183-198

${ }^{7}$ Boschen MJ. Publication trends in individual anxiety disorders: 1980-2005. J Anxiety Disord. 2008; 22:570-575

${ }^{8}$ Boschen MJ, Neumann, DL, Waters, AM. Relapse of successfully treated anxiety and fear: Theoretical issues and recommendations for clinical practice. Aust NZJ Psychiatry 2009; 43:89-100

${ }^{9}$ Yonkers KA, Bruce SE, Dyck IR, Keller MB. Chronicity, relapse, and illness - course of panic disorder, social phobia, and generalized anxiety disorder: Findings in men and women from 8 years of follow-up. Depress Anxiety 2003; 17:173-179 
${ }^{10}$ Baldwin DS, Ajel K. Role of pregabalin in the treatment of generalized anxiety disorder. Neuropsychiatr Dis Treat 2007; 3:185-191

${ }^{11}$ Boschen MJ. Generalized anxiety disorder in adults: Focus on pregabalin. Clin Med Insights: Psychiat 2011; 4:17-35

${ }^{12}$ Czuczwar SJ, Patsalos PM. The new generation of GABA enhancers: Potential in the treatment of epilepsy. CNS Drugs 2001; 15:339-350

${ }^{13}$ Stahl S. Anticonvulsants as anxiolytics, part 2: Pregabalin and gabapentin as $\alpha_{2} \delta$ ligands at voltage-gated calcium channels. J Clin Psychiat 2004; 65:460-461

${ }^{14}$ Stahl S. Mechanism of action of $\alpha_{2} \delta$ ligands: Voltage sensitive calcium channel (VSCC) modulators. J Clin Psychiat 2004; 65:1033-1034

${ }^{15}$ Feltner DE, Crockatt JG, Dubovsky SJ, et al. A randomized, double-blind, placebocontrolled, fixed-dose, multicenter study of pregabalin in patients with generalized anxiety disorder. J Clin Psychopharmacol. 2003; 23: 240-249

${ }^{16}$ Pande AC, Crockatt JG, Feltner DE, et al. Pregabalin in generalized anxiety disorder: A placebo-controlled trial. Am J Psychiat 2003; 160:533-540

${ }^{17}$ Rickels K, Pollack MH, Feltner DE, et al. Pregabalin for treatment of generalized anxiety disorder: A 4-week, multicenter, double-blind, placebo-controlled trial of pregabalin and alprazolam. Arch Gen Psychiat 2005; 62:1022-1030

${ }^{18}$ Pohl RB, Feltner DE, Fieve RR, Pande AC. Efficacy of pregabalin in the treatment of generalized anxiety disorder: Double-blind, plaebo-controlled comparison of BID versus TID dosing. J Clin Psychopharmacol 2005; 25:151-158

${ }^{19}$ Montgomery SA, Tobias K, Zornberg GL, Kasper S, Pande AC. Efficacy and safety of pregabalin in the treatment of generalized anxiety disorder: A 6-week, multicenter, randomized, double-blind, placebo-controlled comparison of pregabalin and venlafaxine. J Clin Psychiat 2006; 67:771-782 
${ }^{20}$ Montgomery SA, Chatamra K, Pauer L, Whalen E, Baldinetti F. Efficacy and safety of pregabalin in elderly people with generalized anxiety disorder. Br J Psychiat $2008 ; 193: 389-394$

${ }^{21}$ Kasper S, Herman B, Nivoli G, et al. Efficacy of pregabalin and venlafaxine-XR in generalized anxiety disorder: Results of a double-blind, placebo-controlled 8week trial. Int Clin Psychopharmacol 2009; 24: 87-96

${ }^{22}$ Feltner D, Wittchen H-U, Kavoussi R, Brock J, Baldinetti F, Pande AC. Long-term efficacy of pregabalin in generalized anxiety disorder. Int Clin Psychopharmacol 2008; 23:18-28

${ }^{23}$ Boschen MJ. A meta-analysis of the efficacy of pregabalin in the treatment of generalized anxiety disorder. Can J Psychiatry; in press

${ }^{24}$ Bech P. Dose-response relationship of pregabalin in patients with generalized anxiety disorder: A pooled analysis of four placebo-controlled trials. Pharmacopsychiatry 2007; 40:163-168

${ }^{25}$ Lydiard RB, Rickels K, Herman B, Feltner DE. Comparative efficacy of pregabalin and benzodiazepines in treating the psychic and somatic symptoms of generalized anxiety disorder. Int J Neuropsychopharmacol 2010; 13:229-241

${ }^{26}$ SPSS Inc. SPSS for Windows, Release 17.0. Chicago: SPSS Inc., 2008.

${ }^{27}$ Montgomery SA, Kasper S. Pharmacotherapy Update: Pregabalin in the treatment of generalized anxiety disorder. Clin Med Insights: Therapeutics 2010; 2:189-202

${ }^{28}$ Frame B, Miller R, Hutmacher MM. Joint modeling of dizziness, drowsiness, and dropout associated with pregabalin and placebo treatment of generalized anxiety disorder. J Pharmacokinet Pharmacodyn 2009; 36: 565-584. 


\section{Financial Disclosure}

The current research has not been funded. The author has no current or previous associations with any pharmaceutical company. There are no other conflicts of interest. 


\section{Figure Captions}

Figure 1. Dose-response curve of effect of pregabalin on Hamilton Anxiety Rating Scale total score.

Figure 2. Dose-response curve of effect of pregabalin on Hamilton Anxiety Rating Scale psychic anxiety score.

Figure 3. Dose-response curve of effect of pregabalin on Hamilton Anxiety Rating Scale somatic anxiety score. 